\title{
Clinical Significance of Platelet Volume and Other Platelet Parameters in Acute Myocardial Infarction and Stable Coronary Artery Disease
}

\author{
Liumei Ding, ${ }^{1 \oplus}$ Lihua Sun, ${ }^{2}$ Feng Wang, ${ }^{1 \oplus}$ Liejun Zhu, ${ }^{1 \oplus}$ Ting Zhang, ${ }^{1 \oplus}$ Fanli Hua ${ }^{2}$ \\ Clinical Laboratory - Jinshan Hospital of Fudan University, ${ }^{1}$ Shanghai - China \\ Department of Hematology - Zhongshan Hospital, ${ }^{2}$ Qingpu Branch, Shanghai - China \\ Liumei Ding and Lihua Sun contributed equally to this work as the first authorship.
}

\section{Abstract}

Background: Platelets are important in the initiation of thrombosis, and their morphological and functional changes are closely related with the occurrence and development of coronary artery thrombosis. Platelet parameters might be valuable in distinguishing between acute myocardial infarction (AMI) and stable coronary artery disease (SCAD).

Objective: This study was designed to detect and compare changes in platelet parameters, such as mean platelet volume (MPV) in patients with acute myocardial infarction (AMI) and stable coronary artery disease (SCAD) and to investigate their roles in these diseases.

Methods: Specimen collection: Between January 2011 and December 2013, $2 \mathrm{~mL}$ of elbow vein blood was drawn from each of 31 patients primarily diagnosed with AMI, 34 SCAD patients and 50 healthy subjects; and placed in EDTA-K2 anticoagulant tubes. Platelet count (PLT), MPV, plateletcrit (PCT), platelet distribution width (PDW), white blood cell (WBC) and neutrophil (NEU) counts were determined using an STKS automated hematology analyzer (Beckman Courter).

Results: Compared with the control group, MPV levels were significantly higher in the AMI and SCAD groups (p < 0.05), while PLT was significantly lower $(p<0.05)$.

Conclusion: These results suggest that MPV and other related parameters have a certain value in the diagnosis of SCAD and AMI. (Arq Bras Cardiol. 2019; 112(6):715-719)

Keywords: Acute Coronary Syndrome/physiopathology; Coronary Thrombosis; Mean Platelet Volume; Myocardial Infarction.

\section{Introduction}

Acute coronary syndromes (ACS) are a group of clinical syndromes, of which pathological bases are coronary atherosclerosis plaques rupture or erosion, ${ }^{1}$ followed by complete or incomplete occlusive thrombosis. ACS include acute myocardial infarction (AMI) and unstable angina pectoris. Among these, AMI refers to acute focal myocardial necrosis caused by prolonged and severe myocardial ischemia. ${ }^{2}$

In 2013, the European Society of Cardiology issued the management guidelines for stable coronary artery disease (SCAD). ${ }^{3}$ These guidelines clearly pointed out that SCAD also include situations of no symptoms or stable symptoms after the stabilization of acute coronary syndrome, besides stable angina pectoris. These situations cannot be clearly distinguished from ACS. Previous studies have revealed that platelet is an important medium in the initiation of thrombosis, and its morphological and functional changes are closely correlated with the occurrence and development of coronary artery thrombosis. ${ }^{4,5}$

Additionally, mean platelet volume (MPV), one of the platelet parameters in $\mathrm{AMI}$, is significantly higher than in normal subjects, hence MPV is considered as a predictor

Mailing Address: Fanli Hua •

№. 1158 of East Gongyuan Road, Qingpu District. 201700, Shanghai - China E-mail: huafanli_cn@163.com

Manuscript received May 21, 2018, revised manuscript September 28, 2018, accepted November 14, 2018

DOI: $10.5935 / a b c .20190058$ for AMI. ${ }^{6}$ However, the value of platelet parameters in distinguishing between AMI and SCAD has not been reported in China. It has been reported that the supersensitive troponin level was elevated in patients with SCAD. ${ }^{7}$ Although it did not reach the threshold for the diagnosis of myocardial infarction, the prognosis was poorer, when compared with SCAD patients without an elevated troponin level. In our study, differences in platelet parameters such as MPV in AMI patients, SCAD patients and healthy subjects were analyzed, and the significance of these parameters for predicting the disease was investigated.

\section{Methods}

\section{General Information}

A total of 31 patients primarily diagnosed with AMI were enrolled in this study. All patients had been diagnosed for the first time, did not undergo anticoagulation or stent percutaneous coronary intervention, and met the international and Chinese guidelines for the diagnosis of AMI. Of these patients, 23 patients were males and eight patients were females; and the mean age of these patients was $64.4 \pm 11.6$ years. Thirty-four patients were diagnosed with SCAD. These patients were required to meet the following criteria: (1) patients diagnosed with AMI for more than two months; (2) patients without pectoralgia; (3) patients who were receiving anticoagulation drug treatment. Of these patients, 28 patients were males and six patients were females; the mean age of these patients was $60.6 \pm 13.1$ years. The control group comprised 50 healthy subjects who underwent physical examinations at the Outpatient Department. 
Among these subjects, 38 were males and 12 were females, and their mean age was $60.9 \pm 6.9$ years.

None of the abovementioned patients had severe liver or kidney disease, malignant tumors, idiopathic thrombocytopenia, or thrombocytopenia caused by other primary diseases.

\section{Sample collection}

In the early morning, $2 \mathrm{~mL}$ of elbow vein blood was withdrawn from each of these subjects under a fasting state, without receiving any hemostasis, coagulation and anticoagulation drugs. The collected blood was placed in EDTA-K2 anticoagulant tubes.

\section{Detection method}

Platelet count (PLT), plateletcrit (PCT), MPV, platelet distribution width (PDW), white blood cell (WBC) and neutrophil (NEU) counts were determined using an STKS automated hematology analyzer (Beckman Courter).

\section{Statistical analysis}

Statistical analysis was performed using the GraphPad Prism 5 software. Measurement data were presented as mean \pm standard deviation $(x \pm S D)$. Comparison of the means in multiple samples was performed using unpaired t-test. $p<0.05$ was considered statistically significant. The prediction value of platelet parameters and the total leukocyte count for $\mathrm{AMI}$ and SCAD were evaluated using the receiver operating characteristic (ROC) curve and the area under the ROC curve (AUC). Correlations between parameters were analyzed using Pearson's correlation statistical method.

\section{Results}

Results of the four platelet parameters and the total WBC count in AMI patients, SCAD patients and normal healthy subjects (Table 1 ).

As shown in Table 1, compared with the control group, the MPV levels were significantly higher $(p<0.01)$, while the PLT and the PDW were significantly lower $(p<0.05)$ in the AMI and SCAD groups. Moreover, compared to the control group, the WBC and NEU levels were also significantly higher $(p<0.01)$ in the $\mathrm{AMI}$ group, but there was no significant difference in the SCAD group. However, the PCT levels had no significant difference between the patient groups and the control group.
Evaluation of the diagnostic efficacy of each index by the diagnostic test

As shown in Figure 1, in the AMI group, the AUC of PLT $(95 \% \mathrm{Cl})$ was $0.6474(0.5206-0.7742)$ and the $p$-value compared with controls was $<0.05$, which shows that the differences were statistically significant. The AUC of MPV $(95 \% \mathrm{Cl})$ was $0.9032(0.8232-0.9832)$ and the $p$-value compared with controls was $<0.01$, which revealed that the differences were statistically significant. Furthermore, the AUC of PDW (95\% Cl) was 0.6529 (0.5239-0.7819) and the p-value compared with controls was < 0.05; the AUC of PCT $(95 \% \mathrm{Cl})$ was $0.5687(0.4364-0.701)$ and the $p$-value compared with controls was $<0.05$, and the AUC of WBC $(95 \% \mathrm{Cl})$ was $0.9190(0.8475-0.9906)$ and the $p$-value compared with controls was $<0.01$. Finally, the AUC of NEU $(95 \% \mathrm{Cl})$ was $0.9310(0.8678-0.9942)$ and the $p$-value compared with controls was $<0.01$. These differences were all statistically significant. These results imply that the diagnostic value of PLT, MPV, WBC and NEU was significantly higher in the AMI group.

As shown in Figure 2, in the SCAD group, the AUC of PLT $(95 \% \mathrm{Cl})$ was $0.6176(0.4907-0.7445)$ and the p-value compared with controls was $>0.05$. The AUC of PDW $(95 \% \mathrm{Cl})$ was $0.6818(0.5554-0.8081)$ and the $p$-value compared with controls was $<0.01$, with the differences being statistically significant. The AUC of PDW $(95 \% \mathrm{Cl})$ was 0.5609 (0.4268-0.6949) and the p-value compared with controls was $>0.05$, whereas the AUC of PCT $(95 \% \mathrm{Cl})$ was $0.5332(0.3994-0.6671)$ and the $p$-value compared with controls was $>0.05$, and the AUC of WBC $(95 \% \mathrm{Cl})$ was $0.5635(0.4368-0.6903)$ and the p-value compared with controls was $>0.05$. Finally, the AUC of $\mathrm{NEU}(95 \% \mathrm{Cl})$ was $0.5447(0.4138-0.6756)$ and the $p$-value compared with controls was $>0.05$. These results imply that the diagnostic value of MPV was significantly higher in the SCAD group.

\section{Correlation analysis of MPV with PLT, PCT and PDW in patients with myocardial infarction}

Pearson's correlation coefficient between MPV and PLT was 0.3817; Pearson's correlation coefficient between MPV and PCT was 0.1103. Pearson's correlation coefficient between MPV and PDW was 0.0726. It indicates that the MPV and PLT in patients with myocardial infarction have a strong correlation.

Table 1 - Platelet parameters and total number of white blood cells and neutrophils in the AMI, SCAD and the control groups

\begin{tabular}{|c|c|c|c|}
\hline Parameters & AMI $(n=31)$ & $\operatorname{SCAD}(n=34)$ & control group $(n=50)$ \\
\hline PLT (x 109) & $185.84 \pm 61.26$ & $193.62 \pm 47.1$ & $206.28 \pm 36.17$ \\
\hline MPV (fL) & $10.42 \pm 1.26$ & $9.16 \pm 1.28$ & $8.42 \pm 0.72$ \\
\hline РCT (\%) & $0.1777 \pm 0.0464$ & $0.1709 \pm 0.0393$ & $0.1693 \pm 0.0287$ \\
\hline PDW (\%) & $16.2 \pm 0.65$ & $16.0 \pm 1.37$ & $16.5 \pm 0.32$ \\
\hline WBC $\left(\times 10^{9} / L\right)$ & $10.60 \pm 2.53$ & $6.61 \pm 1.54$ & $6.37 \pm 1.25$ \\
\hline NEU (x 109/L) & $8.34 \pm 2.66$ & $3.99 \pm 1.25$ & $3.79 \pm 0.93$ \\
\hline
\end{tabular}

Compared with the control group, the MPV levels were significantly higher $(p<0.01)$, while the PLT and the PDW were significantly lower $(p<0.05)$ in the AMI and $S C A D$ groups. Besides, compared to the control group, the WBC and NEU levels were also significantly higher $(p<0.01)$ in the AMI group, but there was no significant difference in the SCAD group. However, the PCT levels showed no significant difference between the patient groups and the control group. 


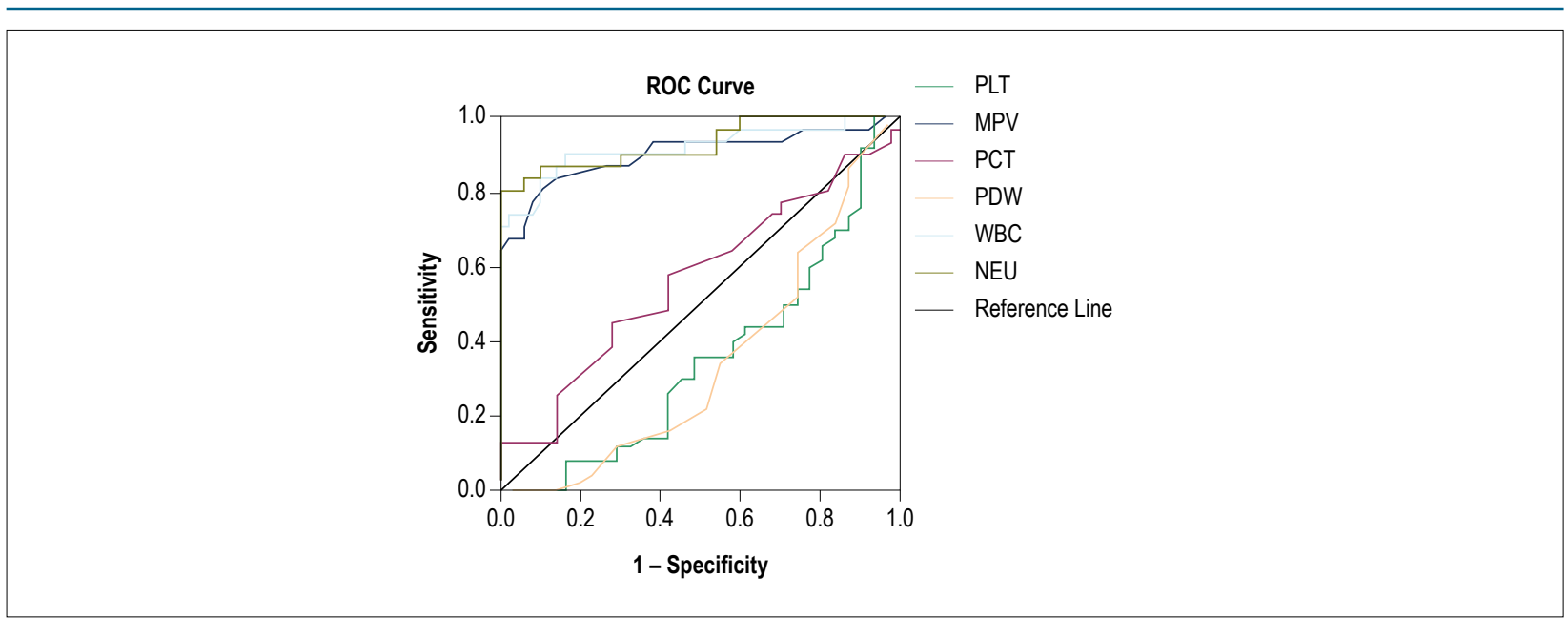

Figure 1 - ROC curves of PLT, PDW, MPV, PCT, WBC and NEU for predicting AMI. PLT: platelet count; MPV: mean platelet volume; PCT: plateletcrit; PDW: platelet distribution width; WBC: white blood cell; NEU: neutrophils; AMI: acute myocardial infarction.

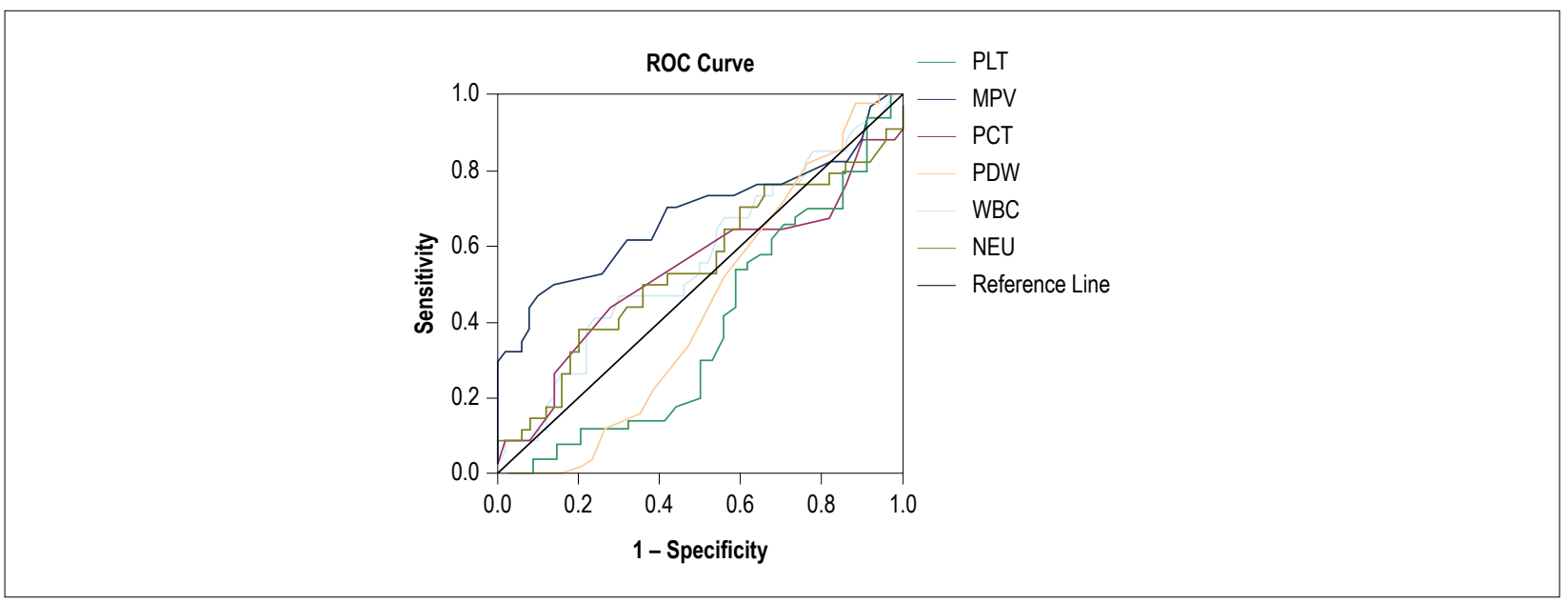

Figure 2 - ROC curves of PLT, PDW, MPV, PCT, WBC and NEU for predicting SCAD. ROC, receiver operating characteristics; PLT: platelet count; MPV: mean platelet volume; PCT: plateletcrit; PDW: platelet distribution width; WBC: white blood cell; NEU: neutrophils; SCAD: stable coronary artery disease.

\section{Discussion}

The key mechanisms of ACS include coronary plaque rupture, platelet aggregation and thrombus formation. Mounting evidence has revealed that inflammation is an important precipitating event in the pathogenesis of ACS. ${ }^{8}$ Inflammatory response plays an important role in the process of the occurrence, development and prognosis of coronary heart diseases. As markers of inflammatory response, WBC and NEU are closely correlated to cardiovascular system diseases. Studies have revealed that both can directly reflect the level of inflammation in coronary artery lesions, thereby further reflecting the severity of coronary artery lesions. ${ }^{9,10}$ Studies have confirmed that the NEU/lymphocyte ratio could serve as a predictor of major adverse cardiovascular events. ${ }^{11-13}$ This study revealed that there was a leukocyte-mediated inflammatory response in patients with $\mathrm{AMI}$ and that serum leukocyte levels were higher in SCAD and AMI patients than in normal healthy subjects. Furthermore, serum NEU levels were significantly abnormal in AMI patients, suggesting that inflammatory response is more intense in AMI patients. Hence, we consider that the reason may be that the range of inflammation is broader in myocardial infarction.

MPV reflects the degree of activation of platelets to a certain extent and is considered an important marker of cardiovascular disease. ${ }^{14}$ Furthermore, it can be used in risk prediction, diagnosis and prognosis assessment of cardiovascular diseases. ${ }^{15-17}$

A recent study revealed that MPV significantly increased in patients with $\mathrm{AMI}$, and the increase in MPV was correlated to the long-term prognosis of AMI patients to a certain extent. ${ }^{18}$ Our study also revealed that MPV was significantly increased in patients in the AMI and SCAD groups. The increase in MPV is likely correlated to the intensity of inflammatory response in the body. 
MPV has important significance in the evaluation of arterial thrombotic diseases such as cardiovascular thrombotic diseases. Therefore, MPV can act as an independent factor for the diagnosis and assessment of conditions and the curative effect of ACS. MPV levels in both the AMI and SCAD groups were significantly higher than normal levels, while PLT levels were significantly lower. These indicators are worthwhile in practice for disease diagnosis. In addition, based on the assessment of the diagnostic efficacy of the ROC curve for these parameters, the predicting value of MPV and PLT in AMI was superior to that in SCAD. In summary, the present study revealed that MPV- and PLT-related indicators are valuable for AMI and SCAD diagnostic prediction. However, there are two shortcomings of our study: one is that the study design is cross-sectional, because of the difficulty in keeping contact with these patients; the other is that there are only about 30 patients in the AMI and SCAD groups. There is no doubt that these two problems constitute limitations of our study. Further studies need to be carried out in a larger cohorts of patients with AMI and SCAD, with a more appropriate design to confirm its value as a diagnostic marker.

\section{Author contributions}

Conception and design of the research: Ding L, Hua F; acquisition of data: Ding L, Sun L, Wang F, Zhu L; analysis and interpretation of the data: Ding L, Sun L, Wang F, Zhu L, Zhang T; statistical analysis: Ding L, Sun L, Wang F, Zhang T, Hua F; writing of the manuscript: Ding L, Zhu L, Zhang T, Hua F; critical revision of the manuscript for intellectual content: Hua F. Supervision: Hua F.

\section{Potential Conflict of Interest}

No potential conflict of interest relevant to this article was reported.

\section{Sources of Funding}

There were no external funding sources for this study.

\section{Study Association}

This study is not associated with any thesis or dissertation work.

\section{Ethics approval and consent to participate}

This study was approved by the Ethics Committee of the Jinshan Hospital, Fudan University under protocol number 785. All the procedures in this study were in accordance with the 1975 Helsinki Declaration, updated in 2013. Informed consent was obtained from all participants included in the study.

\section{References}

1. Tomey M, Mehran R. Dual antiplatelet therapy dilemmas: duration and choice of antiplatelets in acute coronary syndromes. Curr Cardiol Rep. 2013;15(10):405

2. Tjia J, Allison J, Saczynski JS, Tisminetzky M, Givens JL, Lapane K, et al. Encouraging trends in acute myocardial infarction survival in the oldest old. Am J Med. 2013;126(9):798-804.

3. Task Force Members, Montalescot G, Sechtem U, Achenbach S, Andreotti F Arden C, et al. 2013 ESC guidelines on the management of stable coronary artery disease. Eur Heart J. 2013;34(38):2949-3003.

4. Gawaz M. Role of platelets in coronary thrombosis and reperfusion of ischemic myocardium. Cardiovasc Res. 2004;61(3):498-511.

5. Larsen SB, Grove EL, Kristensen SD, Hvas AM. Reduced antiplatelet effect of aspirin is associated with low-grade inflammation in patients with coronary artery disease. Thromb Haemost. 2013;109(5):920-9.

6. Chu SG, Becker RC, Berger PB, Bhatt DL, Eikelboom JW, Konkle B, et al. Mean platelet volume as a predictor of cardiovascular risk: a systematic review and meta-analysis. J Thromb Haemost. 2010;8(1):148-56.

7. Carda R, Aceña Á, Pello A, Cristóbal C, Tarín N, Huelmos A, et al. The prognostic value of high-sensitive troponin i in stable coronary artery disease depends on age and other clinical variables. Cardiology. 2015;132(1):1-8.

8. Liu SW, Qiao SB, Yuan JS, Liu DQ. Association of plasma visfatin levels with inflammation, atherosclerosis and acute coronary syndromes (ACS) in humans. Clin Endocrinol (Oxf). 2009;71(2):202-7.

9. Pearson TA, Mensah GA, Alexander RW, Anderson JL, Cannon RO, Criqui $M$, et al. Markers of inflammation and cardiovascular disease application to clinical and public health practice: a statement for healthcare professionals from the centers for disease control and prevention and the American Heart Association. Circulation. 2003;107(3):499-511.
10. Cannon CP, McCabe CH, Wilcox RG, BentleyJH, Braunwald E. Association of white blood cell count with increased mortality in acute myocardial infarction and unstable angina pectoris. Am J Cardiol. 2001;87(5):636-9, A10.

11. Hong LF, Li XL, Luo SH, Guo YL, Liu J, Zhu CG. Relation of leukocytes and its subsets counts with the severity of stable coronary artery disease in patients with diabetic mellitus. PLoS One. 2014;9(3):e90663.

12. Azab B, Chainani V, Shah N, McGinn JT. Neutrophil-Lymphocyte ratio as a predictor of major adverse cardiac events among diabetic population a 4-year follow-up study. Angiology. 2013;64(6):456-65.

13. Papa A, Emdin M, Passino C, Michelassi C, Battaglia D, Cocci F. Predictive value of elevated neutrophil-lymphocyte ratio on cardiac mortality in patients with stable coronary artery disease. Clin Chim Acta. 2008;395(1-2):27-31.

14. Ihara A, Kawamoto T, Matsumoto K, Shouno S, Hirahara C, Morimoto T, et al. Relationship between platelet indexes and coronary angiographic findings in patients with ischemic heart disease. Pathophysiol Haemost Thromb. 2006;35(5):376-9.

15. Bae MH, Lee JH, Yang DH, Park HS, Cho Y, Chae SC. White blood cell, hemoglobin and platelet distribution width as short-term prognostic markers in patients with acute myocardial infarction. J Korean Med Sci. 2014;29(4):519-26

16. Bath PM, Butterworth RJ. Platelet size: measurement, physiology and vascular disease. Blood Coagul Fibrinolysis. 1996;7(2):157-61.

17. Chu SG, Becker RC, Berger PB, Bhatt DL, Eikelboom JW, Konkle B, et al. Mean platelet volume as a predictor of cardiovascular risk: a systematic review and meta-analysis. J Thromb Haemost. 2010;8(1):148-56.

18. Ranjith MP, DivyaRaj R, Mathew D, George B, Krishnan MN. Mean platelet volume and cardiovascular outcomes in acute myocardial infarction. Heart Asia. 2016;8(1):16-20. 
The clinical significance of platelet parameters such as MPV

Original Article 